\title{
Weak saturation numbers of complete bipartite graphs in the clique
}

\author{
Gal Kronenberg * $\quad$ Taísa Martins ${ }^{\dagger} \quad$ Natasha Morrison ${ }^{\ddagger}$
}

\begin{abstract}
The notion of weak saturation was introduced by Bollobás in 1968. Let $F$ and $H$ be graphs. A spanning subgraph $G \subseteq F$ is weakly $(F, H)$-saturated if it contains no copy of $H$ but there exists an ordering $e_{1}, \ldots, e_{t}$ of $E(F) \backslash E(G)$ such that for each $i \in[t]$, the graph $G \cup\left\{e_{1}, \ldots, e_{i}\right\}$ contains a copy $H^{\prime}$ of $H$ such that $e_{i} \in H^{\prime}$. Define wsat $(F, H)$ to be the minimum number of edges in a weakly $(F, H)$-saturated graph. In this paper, we prove for all $t \geq 2$ and $n \geq 3 t-3$, that $\operatorname{wsat}\left(K_{n}, K_{t, t}\right)=(t-1)(n+1-t / 2)$, and we determine the value of wsat $\left(K_{n}, K_{t-1, t}\right)$ as well. For fixed $2 \leq s<t$, we also obtain bounds on $\operatorname{wsat}\left(K_{n}, K_{s, t}\right)$ that are asymptotically tight.
\end{abstract}

\section{Introduction}

Let $F$ and $H$ be graphs. A spanning subgraph $G$ of $F$ is said to be weakly $(F, H)$-saturated, if $G$ contains no copies of $H$, but there exists an ordering $e_{1}, \ldots, e_{t}$ of $E(F) \backslash E(G)$ such that the addition of $e_{i}$ to $G \cup\left\{e_{1}, \ldots, e_{i-1}\right\}$ creates a new copy $H^{\prime}$ of $H$ where $e_{i} \in H^{\prime}$, for every $i \in[t]$. The weak saturation number of $H$ in $F$, is defined to be

$$
\operatorname{wsat}(F, H):=\min \{|E(G)|: G \text { is weakly }(F, H) \text {-saturated }\}
$$

That is, wsat $(F, H)$ is the minimum number of edges of a weakly $(F, H)$-saturated graph. In the most natural case where $F$ is the complete graph on $n$ vertices, denoted $K_{n}$, we write $\operatorname{wsat}(n, H):=$ $\operatorname{wsat}\left(K_{n}, H\right)$.

Weak saturation of graphs was initially introduced by Bollobás [5] in 1968 and has grown to a substantial area of research. Originally motivated by the problem of determining the saturation ${ }^{1}$ number of $k$-uniform hypergraphs, Bollobás determined wsat $\left(n, K_{m}\right)$ for $3 \leq m \leq 7$ and conjectured

\footnotetext{
*Mathematical Institute, University of Oxford, Oxford, UK. E-mail: kronenberg@maths.ox.ac.uk.

${ }^{\dagger}$ Instituto de Matemática, Universidade Federal Fluminense, Niterói, Brazil.

E-mail: tlmartins@id.uff.br.

${ }^{\ddagger}$ Department of Mathematics and Statistics, University of Victoria, David Turpin Building, 3800 Finnerty Road, Victoria, B.C., Canada V8P $5 \mathrm{C} 2$.

E-mail: nmorrison@uvic.ca.

${ }^{1}$ A spanning subgraph $G \subseteq F$ is $(F, H)$-saturated, if it contains no copy of $H$ but the addition of any edge of $E(F) \backslash E(G)$ creates a copy of $H$.
} 
that the graph obtained by removing a copy of $K_{n-r+2}$ from $K_{n}$ is best possible for wsat $\left(n, K_{r}\right)$. Using a very elegant generalisation of the Bollobás two families theorem [4], Lovász [14] was the first to confirm this conjecture.

Theorem 1 (Lovász [14]). Let $n \geq r \geq 2$. Then

$$
\operatorname{wsat}\left(n, K_{r}\right)=\left(\begin{array}{l}
n \\
2
\end{array}\right)-\left(\begin{array}{c}
n-r+2 \\
2
\end{array}\right)
$$

Independent proofs were later found by Alon [1], Frankl [11, and Kalai [12, 13]. Interestingly, all these proofs utilise algebraic techniques and no combinatorial proof of Theorem 1 is known.

Let $K_{s, t}$ denote the complete bipartite graph with vertex classes size $s$ and $t$. In this article, we study the next most natural question to consider regarding weak saturation: What is wsat $\left(n, K_{s, t}\right)$ ? In the case of the balanced complete bipartite graph we determine this number exactly. Our first theorem is the following.

Theorem 2. Let $t \geq 2$ and $n \geq 3 t-3$. Then

$$
\operatorname{wsat}\left(K_{n}, K_{t, t}\right)=(t-1)(n+1-t / 2) .
$$

Given this theorem, a short argument yields an exact result for $K_{t, t+1}$.

Corollary 3. Let $t \geq 2$ and $n \geq 3 t-3$. Then

$$
\operatorname{wsat}\left(K_{n}, K_{t, t+1}\right)=(t-1)(n+1-t / 2)+1 .
$$

For when $s<t$, we also obtain a general bound for $K_{s, t}$, which is tight asymptotically for fixed $s, t$ and $n$ large.

Theorem 4. Let $2 \leq s<t$ and $n \geq 4 t$. Then

$$
\operatorname{wsat}\left(n, K_{s, t}\right)=n(s-1)+c(s, t)
$$

where $c(s, t)$ is an integer depending only on $s$ and $t$.

Surprisingly, despite the large body of work and number of alternate proofs to determine $\operatorname{wsat}\left(n, K_{m}\right)$, prior to this work very little was known about $\operatorname{wsat}\left(n, K_{s, t}\right)$. The case when $s=2$ and $t=3$, was shown to be $n+1$, by Faudree, Gould and Jacobson [10], who also determined the weak saturation number for various families of sparse graphs. A trivial lower bound of $n \cdot(s-1) / 2$ can be obtained by observing that every vertex in a weakly $\left(K_{n}, K_{s, t}\right)$-saturated graph must have degree at least $(s-1)$, when $s \leq t$. But other than this, no general lower bound was previously known.

A more well studied setting is where the weak saturation process takes place inside a bipartite ambient graph (i.e. $H$ is bipartite). In [1], Alon studied a labelled version of this problem called bisaturation. For a bipartite graph $H=\left(V_{1} \cup V_{2}, E\right)$, we say that a spanning subgraph $G \subseteq K_{\ell, m}$ is weakly $\left(K_{\ell, m}, H\right)$-bisaturated, if there exists an ordering $e_{1}, \ldots, e_{t}$ of $E\left(K_{\ell, m}\right) \backslash E(G)$ such that the addition of $e_{i}$ to $G \cup\left\{e_{1}, \ldots, e_{i-1}\right\}$ will create a new copy $H^{\prime}$ of $H$ where $e_{i} \in H^{\prime}$, with $V_{1}$ 
in the first class and $V_{2}$ in the second class, for every $i \in[t]$. The bisaturation number, denoted $w(\ell, m, H)$, is the minimal possible number of edges in a weakly $H$-bisaturated graph.

The bisaturation number is closely related to the weak saturation number inside a bipartite graph. Indeed, as every weakly $\left(K_{\ell, m}, H\right)$-bisaturated graph $G$ is also weakly $\left(K_{\ell, m}, H\right)$-saturated, we have $w(\ell, m, H) \geq \operatorname{wsat}\left(K_{\ell, m}, H\right)$. In addition, when $s<t$ we also have $w\left(s, n-s, K_{s, t}\right)=$ $\operatorname{wsat}\left(K_{s, n-s}, K_{s, t}\right)$, and when $\ell, m \geq t$, we have $w\left(\ell, m, K_{t, t}\right)=\operatorname{wsat}\left(K_{\ell, m}, K_{t, t}\right)$. The value of $w\left(\ell, m, K_{r, t}\right)$ was determined precisely by Alon, who also proved a generalization for hypergraphs.

Theorem 5 (Alon [1]). For $2 \leq s \leq t$ and $2 \leq \ell \leq m$, we have

$$
w\left(\ell, m, K_{s, t}\right)=\ell \cdot m-(\ell-s+1)(m-t+1)
$$

Moshkovitz and Shapira [17] showed how to deduce wsat $\left(K_{n, n}, K_{s, t}\right)$ from this result, giving the following theorem.

Theorem 6 (Moshkovitz and Shapira [17]). Let $2 \leq s \leq t \leq n$. Then

$$
\operatorname{wsat}\left(K_{n, n}, K_{s, t}\right)=n^{2}-(n-s+1)^{2}+(t-s)^{2} .
$$

We would like to note that the main contribution in [17] is studying an analogous process in multipartite hypergraphs. In doing so, they also prove a very beautiful two-families type theorem.

The proof of Theorem 6 can be easily generalised to determine $\operatorname{wsat}\left(K_{\ell, m}, K_{s, t}\right)$.

Theorem 7. Let $2 \leq s \leq \ell, t \leq m$. Then

$$
\operatorname{wsat}\left(K_{\ell, m}, K_{s, t}\right)=(m+\ell-s+1)(s-1)+(t-s)^{2} .
$$

For completeness, we include the full argument in Appendix A. Observe that when $\ell+m=n$, Theorem 7 implies that $\operatorname{wsat}\left(K_{\ell, m}, K_{t, t}\right)=(t-1)(n+1-t)$. We note that this, along with Theorem 2 gives the following relationship between weak saturation numbers of complete balanced bipartite graphs in the clique and in complete bipartite graphs.

Corollary 8. For $t \geq 2, n \geq 3 t-3$ and $\ell, m \geq 2$ such that $\ell+m=n$, we have

$$
\operatorname{wsat}\left(n, K_{t, t}\right)=\operatorname{wsat}\left(K_{\ell, m}, K_{t, t}\right)+\left(\begin{array}{l}
t \\
2
\end{array}\right) .
$$

In particular, taking a construction of minimum size for wsat $\left(K_{t, n-t}, K_{t, t}\right)$ (see Appendix A) and adding a copy of $K_{t}$ in the larger part of the graph, will give a minimum example for wsat $\left(n, K_{t, t}\right)$. This argument will give an upper bound also for the unbalanced case, that is, the upper bound $\operatorname{wsat}\left(n, K_{s, t}\right) \leq \operatorname{wsat}\left(K_{\ell, m}, K_{s, t}\right)+\left(\begin{array}{l}t \\ 2\end{array}\right)$ is always true for $m+\ell=n$, however it is tight only for the balanced case. Indeed, for $s<t$, the proof of Theorem 4 (more specifically, Proposition 14) will give a better upper bound.

Weak saturation numbers have been also studied in a large number of other settings. Amongst others are the weak saturation numbers for complete multipartite graphs and hypergraphs [1], asymptotics of the weak saturation number of hypergraphs [22], the weak saturation number for 
families of hypergraphs with a fixed number of edges [7, 18, 23, complete bipartite hypergraphs (in complete bipartite hypergraphs) [2, 17, pyramids in hypergraphs [19], families of graphs in the complete graph [20, 6, 21], families of disjoint copies of graphs [9], and the case that $H$ is the hypercube or the grid [2, 3, 16]. For a short survey see [8, Section 10].

The paper is organized as follows. In Section 2 we introduce an important tool that will be used to prove the lower bound in Theorem 2, which is then proved in Section 3 . Theorem 4 is proved in Section 4. We conclude in Section 5 by discussing some generalisations and interesting open problems.

Note added after publication: It was recently brought to our attention that Theorem 2 was independently proved in 1985 by Kalai [12, Theorem 9.1] using matroids.

\section{Preliminaries}

In order to exactly determine a particular weak saturation number, a common strategy is to prove matching upper and lower bounds (often in very different ways). To prove an upper bound of $M$, it suffices to find a construction of a graph with $M$ edges that is weakly $(F, H)$-saturated. Indeed, this is precisely what we do in the proof of Theorem 2 (see Lemma 10). However, in order to prove a lower bound of $M$, we must show that no graph on $M-1$ edges can be weakly $(F, H)$-saturated.

In order to do this we will utilise the following lemma.

Lemma 9 (Balogh, Bollobás, Morris and Riordan [2]). Let $F$ and $H$ be graphs and let $W$ be $a$ vector space. Suppose that there exists a set $\left\{f_{e}: e \in E(F)\right\} \subseteq W$ such that for every copy $H^{\prime}$ of $H$ in $F$ there are non-zero scalars $\left\{c_{e, H^{\prime}}: e \in E\left(H^{\prime}\right)\right\}$ such that $\sum_{e \in E\left(H^{\prime}\right)} c_{e, H^{\prime}} f_{e}=0$. Then

$$
\operatorname{wsat}(F, H) \geq \operatorname{dim}\left(\operatorname{span}\left\{f_{e}: e \in E(F)\right\}\right)
$$

The proof of Lemma 9 is short and beautiful, and so we include it here for the reader's enjoyment.

Proof. Let $F_{0}$ be any weakly $(F, H)$-saturated graph. Let $e_{1}, \ldots, e_{m}$ be an ordering of $E(F) \backslash E\left(F_{0}\right)$ such that for each $i \in[m]$, the graph $F_{i}:=F_{0} \cup\left\{e_{1}, \ldots, e_{i}\right\}$ contains a copy $H_{i}$ of $H$, such that $e_{i} \in H_{i}$. Now, by hypothesis, for each $i \in[m]$, we have

$$
f_{e_{i}} \in \operatorname{span}\left\{f_{e}: e \in E\left(H_{i}\right) \backslash\left\{e_{i}\right\}\right\} .
$$

As $H_{i} \subseteq F_{0} \cup\left\{e_{1}, \ldots, e_{i}\right\}$, this implies that, for each $i$,

$$
f_{e_{i}} \in \operatorname{span}\left\{f_{e}: e \in E\left(F_{0}\right) \cup\left\{e_{1}, \ldots, e_{i-1}\right\}\right\} .
$$

So, for all $i \in[m]$ we have

$$
\operatorname{span}\left\{f_{e}: e \in E\left(F_{i-1}\right)\right\}=\operatorname{span}\left\{f_{e}: e \in E\left(F_{i}\right)\right\} .
$$


And so

$$
\left|E\left(F_{0}\right)\right| \geq \operatorname{dim}\left(\operatorname{span}\left\{f_{e}: e \in E\left(F_{0}\right)\right\}\right)=\operatorname{dim}\left(\operatorname{span}\left\{f_{e}: e \in E\left(F_{m}\right)\right\}\right)=\operatorname{dim}\left(\operatorname{span}\left\{f_{e}: e \in F\right\}\right),
$$

as required.

We would like to briefly remark that the condition in the lemma of assigning vectors to edges in such a way that those on copies of $H$ satisfy a particular dependence is not in itself difficult to satisfy (for example, just put the same vector on every edge). However, doing this would result in a terrible lower bound on wsat $(F, H)\left(\operatorname{as} \operatorname{dim}\left(\operatorname{span}\left\{f_{e}: e \in E(F)\right\}=1\right)\right)$. So the difficulty in applying this lemma lies in finding vectors that both satisfy the dependence condition and have a large span.

This lemma is very powerful, as it turns the problem of finding a lower bound for a weak saturation number into a constructive problem. Indeed, one need only find a suitable vector space and assign certain vectors to the edges of $F$ to obtain the bound. In Lemma 11, we present a collection of vectors such that the vectors assigned to each copy of $K_{t, t}$ satisfy the required dependence property. We then show that the vectors assigned to a copy of our upper bound construction are linearly independent (and hence the lower bound matches the upper).

A more general version of Lemma 9 was originally used by Balogh, Bollobás, Morris and Riordan [2] in the study of a bootstrap process on hypergraphs. It has since been used to determine exact weak saturation numbers (see for example [15, 16]). We remark that in all these cases, and in the case of Theorem 2 here, no purely combinatorial proof is known that gives any of the lower bounds that are proved via Lemma 9. This is a common phenomenon in this area, where the literature is full of proofs via algebraic techniques for which no combinatorial proof is known. It would be very interesting to see a purely combinatorial proof of any of these weak saturation results.

\section{Proof of Theorem 2}

We will prove Theorem 2 in two steps. First in Lemma 10 we will prove the upper bound by exhibiting a weakly $\left(K_{n}, K_{t, t}\right)$-saturated graph with $(t-1)(n+1-t / 2)$ edges. Then in Lemma 11 we prove a matching lower bound by applying Lemma 9 .

Let $G$ be a graph. For disjoint vertex sets $X, Y \subseteq V(G)$, we denote by $G[X, Y]$ the bipartite graph induced by the edges of $G$ with one endpoint in $X$ and the other in $Y$.

Construction 1. Let $X, Y$ and $Z$ be disjoint subsets of $[n]$ of cardinality $t, t-1$ and $n-2 t+1$, respectively. Define $G_{n}$ to be the graph on vertex set $X \cup Y \cup Z$ and $u v$ is an edge of $G_{n}$ if and only if either $u \in X \cup Z$ and $v \in Y$, or $u, v \in X$. That is, $X$ is a clique on $t$ vertices, $Y$ and $Z$ are both independent sets, and $G_{n}[X \cup Z, Y]$ is a complete bipartite graph.

See Figure 1 for an illustration. Observe that $G_{n}$ has $(t-1)(n+1-t / 2)$ edges. To prove the upper bound we will show that $G_{n}$ is weakly $\left(K_{n}, K_{t, t}\right)$-saturated.

Lemma 10. Let $t \geq 2$ and $n \geq 3 t-3$. Then $\operatorname{wsat}\left(K_{n}, K_{t, t}\right) \leq(t-1)(n+1-t / 2)$. 


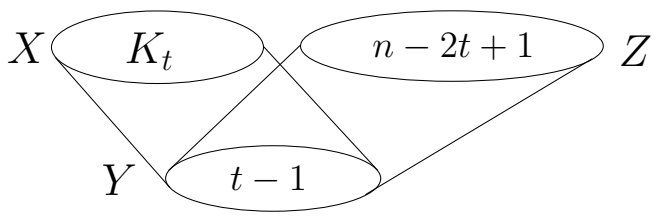

Figure 1: The graph $G_{n}$. The sets $Y$ and $Z$ are independent sets of cardinality $t-1$ and $n-2 t+1$, respectively. All edges between $X \cup Z$ and $Y$ are present. $X$ is a clique on $t$ vertices.

Proof. We will show that $G_{n}$ is weakly $\left(K_{n}, K_{t, t}\right)$-saturated by adding the edges of $E\left(K_{n}\right) \backslash E\left(G_{n}\right)$ in such a way that the addition of each edge $e$ creates a copy of $K_{t, t}$ containing $e$.

Let $X, Y, Z$ be as defined above. We are first able to add all the edges between $X$ and $Z$ (in any order). Indeed, consider $e=x z$ for some $x \in X$ and $z \in Z$. Observe that $G_{n}[Y \cup\{x\}, X \backslash\{x\} \cup\{z\}]$ is a copy of $K_{t, t} \backslash\{e\}$ and hence $e$ can be added.

We next show that we can add all edges within $Z$. Let $e=z_{1} z_{2}$, where $z_{1}, z_{2} \in Z$ and let $X^{\prime}$ be a subset of $X$ of size $t-1$. Observe that $G_{n}\left[Y \cup\left\{z_{1}\right\}, X^{\prime} \cup\left\{z_{2}\right\}\right]$ is a copy of $K_{t, t} \backslash\{e\}$. Hence $e$ can be added.

It remains to show that any edge $e=y_{1} y_{2}$, where $y_{1}, y_{2} \in Y$ can be added. As all edges outside of $Y$ have been added and $n-t+1 \geq 2 t-2$, any subset $S$ of $X \cup Z$ of cardinality $2 t-2$ along with $y_{1}$ and $y_{2}$ contains a copy of $K_{t, t} \backslash\{e\}$. Hence every edge in $Y$ can be added. This completes the proof.

Proving the lower bound is much more involved. Our strategy is to apply Lemma 9. We will construct a family of vectors $\left\{f_{e}: e \in E\left(K_{n}\right)\right\}$ such that: (1) for any copy $H$ of $K_{t, t}$ in $K_{n}$, the vectors $\left\{f_{e}: e \in E(H)\right\}$ have a non-trivial dependence; $(2)$ the subset of vectors $\left\{f_{e}: e \in E\left(G_{n}\right)\right\}$ are linearly independent.

Lemma 11. Let $t \geq 2$ and $n \geq 3 t-3$. Then $\operatorname{wsat}\left(K_{n}, K_{t, t}\right) \geq(t-1)(n+1-t / 2)$.

Proof. Let $W$ be a vector space that is the direct sum of $n$ copies of $\mathbb{R}^{t-1}$, one for each vertex of $K_{n}$. That is,

$$
W:=\bigoplus_{v \in K_{n}} \mathbb{R}^{t-1}
$$

For each edge $e \in K_{n}$ we will associate a vector $f_{e} \in W$, in such a way that the hypotheses of Lemma 9 are satisfied.

For $v \in K_{n}$ and $w \in W$, let $\pi_{v}: W \rightarrow \mathbb{R}^{t-1}$, denote the projection of $w$ to the copy of $\mathbb{R}^{t-1}$ corresponding to $v$. Let $U=\left\{\mathbf{u}_{v}: v \in K_{n}\right\} \subseteq \mathbb{R}^{t-1}$ be a family of $n$ vectors in general position. That is, any $t-1$ vectors of $U$ are linearly independent (and hence any $t$ vectors have a unique dependence, up to scaling by a constant factor). Existence of such a family can be seen by picking $n$ random vectors from $\mathbb{R}^{t-1}$ and observing that, with high probability, any subset of size $t-1$ is independent.

To an edge $e=x y \in E\left(K_{n}\right)$ we will associate the vector $f_{e} \in \mathbb{R}^{n(t-1)}$, defined such that $\pi_{x}\left(f_{e}\right)=\mathbf{u}_{y}, \pi_{y}\left(f_{e}\right)=\mathbf{u}_{x}$ and $\pi_{v}\left(f_{e}\right)=0^{t-1}$ (where we use this notation to represent the $(t-1$ )dimensional all zero vector), for all $v \in V\left(K_{n}\right) \backslash\{x, y\}$. Let $E \subseteq E\left(K_{n}\right)$ and $\left\{c_{e}: e \in E\right\}$ be a set 
of non-zero scalars. Observe that

$$
\sum_{e \in E} c_{e} f_{e}=0 \text { if and only if } \pi_{v}\left(\sum_{e \in E} c_{e} f_{e}\right)=\sum_{e \in E} c_{e} \pi_{v}\left(f_{e}\right)=0 \text {, for every } v \in K_{n} .
$$

Let us now affirm that the family $\left\{f_{e}: e \in E\left(K_{n}\right)\right\}$ satisfies the hypotheses of Lemma 9 .

Claim 12. For every copy $H$ of $K_{t, t}$ in $K_{n}$, there exist non-zero scalars $\left\{c_{e, H}: e \in E(H)\right\}$ such that $\sum_{e \in E(H)} c_{e, H} f_{e}=0$.

Proof. For simplicity of notation, we will write $c_{e}$ for $c_{e, H}$. Assume without loss of generality that $V(H)=\left(\left\{v_{1}, \ldots, v_{t}\right\},\left\{w_{1}, \ldots, w_{t}\right\}\right)$. As $U$ is a family of vectors in general position, there exist non-zero scalars $\alpha_{1} \ldots \alpha_{t}$ such that $\sum_{i=1}^{t} \alpha_{i} \mathbf{u}_{v_{i}}=0$. Similarly, let $\beta_{1} \ldots \beta_{t}$ be non-zero scalars such that $\sum_{i=1}^{t} \beta_{i} \mathbf{u}_{w_{i}}=0$. For $e=v_{i} w_{j} \in H$, define $c_{e}:=\alpha_{i} \beta_{j}$. We will show that $\sum_{e \in E(H)} c_{e} f_{e}=0$.

By (1) it suffices to show that, for each $v \in V(H)$, we have $\sum_{e \in E(H)} c_{e} \pi_{v}\left(f_{e}\right)=0$. Without loss of generality, consider $v_{i} \in V(H)$. We have

$$
\sum_{e \in E(H)} c_{e} \pi_{v_{i}}\left(f_{e}\right)=\sum_{j=1}^{t} \alpha_{i} \beta_{j} \mathbf{u}_{w_{j}}=\alpha_{i} \sum_{j=1}^{t} \beta_{j} \mathbf{u}_{w_{j}}=0
$$

by choice of the scalars $\beta_{1}, \ldots, \beta_{t}$. This concludes the proof of the claim.

We will now bound the dimension of the space spanned by the vectors of $\left\{f_{e}: e \in E\left(K_{n}\right)\right\}$.

Claim 13. $\operatorname{dim}\left(\operatorname{span}\left(\left\{f_{e}: e \in E\left(K_{n}\right)\right\}\right)\right) \geq(t-1)(n+1-t / 2)$.

Proof. Recall the definition of the graph $G_{n}$ from Construction 1. We will show that the family of vectors $\left\{f_{e}: e \in G_{n}\right\}$ are linearly independent. As $\left|E\left(G_{n}\right)\right|=(t-1)(n+1-t / 2)$, this will prove the claim.

Let us suppose that $\Sigma:=\sum_{e \in E\left(G_{n}\right)} c_{e} f_{e}=0$. We will show that $c_{e}=0$ for every $e \in E\left(G_{n}\right)$. Recall that $V\left(G_{n}\right)=X \cup Y \cup Z$. First, for $z \in Z$ consider $\pi_{z}(\Sigma)$. Using (1), we have

$$
\pi_{z}\left(\sum_{e \in E\left(G_{n}\right)} c_{e} f_{e}\right)=\sum_{e \in E\left(G_{n}\right)} c_{e} \pi_{z}\left(f_{e}\right)=\sum_{y \in Y} c_{y z} \mathbf{u}_{y}=0 .
$$

As $|Y|=t-1$ and since any $t-1$ vectors of $U$ are linearly independent, $c_{y z}=0$ for all $y \in Y$ and $z \in Z$.

Now suppose, in order to obtain a contradiction, that there exists some $y^{*} \in Y$ and $x^{*} \in X$ such that $c_{y^{*} x^{*}} \neq 0$. Using (1), for each $y \in Y$ we have

$$
0=\sum_{e \in G_{n}} c_{e} \pi_{y}\left(f_{e}\right)=\sum_{x \in X} c_{y x} \mathbf{u}_{x}
$$

In this case, as $|X|=t$ and any $t$ vectors of $U$ are minimally dependent, using (2) we obtain that

$$
c_{y^{*} x} \neq 0, \quad \text { for all } \quad x \in X
$$


In addition, as the dependence of the vectors $\mathcal{X}:=\left\{\mathbf{u}_{x}: x \in X\right\}$ is unique up to scaling by a constant factor, we obtain that for each $y \in Y \backslash\left\{y^{*}\right\}$, there exists $\gamma_{y} \in \mathbb{R}$ such that $c_{y x}=\gamma_{y} c_{y^{*} x}$, for all $x \in X$ (note that $\gamma_{y}$ may be equal to 0 ).

Now consider $\pi_{x}(\Sigma)$, for each $x \in X$. Expanding this out, we obtain

$$
\pi_{x}(\Sigma)=\sum_{y \in Y} c_{y x} \mathbf{u}_{y}+\sum_{x^{\prime} \in X \backslash\{x\}} c_{x x^{\prime}} \mathbf{u}_{x^{\prime}}=c_{y^{*} x}\left(\sum_{y \in Y} \gamma_{y} \mathbf{u}_{y}\right)+\sum_{x^{\prime} \in X \backslash\{x\}} c_{x x^{\prime}} \mathbf{u}_{x^{\prime}} .
$$

Then for each $x_{1}, x_{2} \in X$, we have

$$
\frac{\pi_{x_{1}}(\Sigma)}{c_{y^{*} x_{1}}}-\frac{\pi_{x_{2}}(\Sigma)}{c_{y^{*} x_{2}}}=c_{x_{1} x_{2}}\left(\frac{\mathbf{u}_{x_{2}}}{c_{y^{*} x_{1}}}-\frac{\mathbf{u}_{x_{1}}}{c_{y^{*} x_{2}}}\right)+\sum_{x \in X \backslash\left\{x_{1}, x_{2}\right\}}\left(\frac{c_{x_{1} x}}{c_{y^{*} x_{1}}}-\frac{c_{x_{2} x}}{c_{y^{*} x_{2}}}\right) \mathbf{u}_{x}=0,
$$

as by (1), $\pi_{x}(\Sigma)=0$, for all $x \in X$. The expression (4) is a linear combination of vectors of $\mathcal{X} \subseteq U$, which by definition are minimally dependent as $|\mathcal{X}|=t$. So this dependence is equal (up to a constant scaling factor) to the dependence between the vectors of $\mathcal{X}$ in (2). Hence there exists $\eta \in \mathbb{R}$ such that for all $x \in X$, the coefficient of $\mathbf{u}_{x}$ in (4) is equal to $\eta c_{y^{*} x}$. Therefore, by looking at the coefficients of $\mathbf{u}_{x_{1}}$ and $\mathbf{u}_{x_{2}}$, we obtain that

$$
-\frac{c_{x_{1} x_{2}}}{c_{y^{*} x_{2}}}=\eta c_{y^{*} x_{1}} \quad \text { and } \quad \frac{c_{x_{1} x_{2}}}{c_{y^{*} x_{1}}}=\eta c_{y^{*} x_{2}}
$$

which implies that $c_{x_{1} x_{2}}=0\left(\right.$ as $c_{y^{*} x_{1}} c_{y^{*} x_{2}} \neq 0$, by $\left.(3)\right)$.

But now, for any $x \in X, \pi_{x}(\Sigma)$ is a linear combination of $t-1$ vectors of $U$, and hence $c_{x y}=0$, for all $x \in X, y \in Y$ (using (1) and the fact that any $t-1$ vectors of $U$ are linearly independent). This contradicts our assumption that $c_{y^{*} x^{*}} \neq 0$.

Hence $c_{x y}=0$ for all $x \in X$ and $y \in Y$ and it remains to show that $c_{x x^{\prime}}=0$, for all $x, x^{\prime} \in X$. But now, for any $x \in X$,

$$
\pi_{x}(\Sigma)=\sum_{x^{\prime} \in X \backslash\{x\}} c_{x x^{\prime}} \mathbf{u}_{x^{\prime}}=0,
$$

by (1). As vectors in $U$ are in general position in $\mathbb{R}^{t-1}$, any $t-1$ are linearly independent and hence this expression can only hold if $c_{x x^{\prime}}=0$ for all $x^{\prime} \in X \backslash\{x\}$.

This completes the proof that $\left\{f_{e}: e \in E\left(G_{n}\right)\right\}$ is a family of linearly independent vectors. Hence, $\operatorname{dim}\left(\operatorname{span}\left\{f_{e}: e \in E(F)\right\}\right) \geq|E|=(t-1)(n+1-t / 2)$.

Given Claims 12 and 13, we may apply Lemma 9. This completes the proof of the lemma.

Proof of Theorem 2. The theorem follows immediately from Lemmas 10 and 11.

\subsection{Determining wsat $\left(n, K_{t, t+1}\right)$}

Proof of Corollary 3. For the lower bound, we will show that every graph $G$ which is weakly $\left(n, K_{t, t+1}\right)$-saturated, contains a proper subgraph $G^{\prime}$ which is weakly $\left(n, K_{t, t}\right)$-saturated, and therefore $\operatorname{wsat}\left(n, K_{t, t+1}\right)>\operatorname{wsat}\left(n, K_{t, t}\right)$. Indeed, let $e_{1}, \ldots, e_{t}$ be an ordering of $E\left(K_{n}\right) \backslash E(G)$ such that the addition of $e_{i}$ to $G \cup\left\{e_{1}, \ldots, e_{i-1}\right\}$ creates a copy $H_{i}$ of $K_{t, t+1}$, such that $e_{i} \in H_{i}$. Note that 
this implies that the addition of each edge also creates a copy $H_{i}^{\prime}$ of $K_{t, t}$ with $e_{i} \in H_{i}^{\prime}$. Observe that at the start of the process, $E\left(H_{1}\right) \backslash\left\{e_{1}\right\} \subseteq E(G)$. Therefore $G$ contains a copy of $K_{t, t+1} \backslash\{e\}$, for some edge $e$. In particular, $G$ contains a copy of $K_{t, t}$. So there exists a weakly $\left(K_{n}, K_{t, t}\right)$-saturated subgraph $G^{\prime} \subseteq G$, where $\left|E\left(G^{\prime}\right)\right| \leq|E(G)|-1$.

For the upper bound, we will construct a weakly $\left(K_{n}, K_{t, t+1}\right)$-saturated graph $F_{n}$ with $(t-$ $1)(n+1-t / 2)+1$ edges. Let $X, Y \cup\left\{y^{*}\right\}$ and $Z$ be disjoint sets of vertices of cardinality $t, t$ and $n-2 t$, respectively. Define $F_{n}$ to be the graph on vertex set $X \cup Y \cup Z \cup\left\{y^{*}\right\}$ and $u v$ is an edge of $F_{n}$ if and only if either $u \in X$ and $v \in Y \cup\left\{y^{*}\right\}, u \in Z$ and $v \in Y$ or $u, v \in X$. That is, $X$ is a clique on $t$ vertices, $Y \cup\left\{y^{*}\right\}$ and $Z$ are both independent sets, and $F_{n}(X \cup Z, Y)$ is a complete bipartite graph. Observe that $F_{n}$ has $(t-1)(n+1-t / 2)+1$ edges. It is easy to check that $F_{n}$ is weakly $\left(K_{n}, K_{t, t+1}\right)$ saturated: first add the edges from $y^{*}$ to $Z$, then add the edges between $X$ and $Z$, then the edges within $Z$ and finally the edges within $Y \cup\left\{y^{*}\right\}$.

\section{Proof of Theorem 4}

In this section we prove Theorem 4 4 i.e., we asymptotically determine the value of wsat $\left(n, K_{s, t}\right)$ whenever $2 \leq s<t$ and $n \geq 4 t$. We start by describing a construction to give an upper bound for $\operatorname{wsat}\left(K_{n}, K_{s, t}\right)$ in the spirit of Construction 1 .

Construction 2. We define $V\left(H_{n}\right)=X \cup\left\{x^{*}\right\} \cup Y_{1} \cup Y_{2} \cup W \cup Z$, where $X, Y_{1}, Y_{2}, W$ and $Z$ are disjoint subsets of $[n]$ of cardinality $s-1, t-s, s-1, s-1$ and $n-t-2 s+2$, respectively. As $s<t$, note that $Y_{1} \neq \emptyset$. Let $Y:=Y_{1} \cup Y_{2}$. We have that $u v$ is an edge of $H_{n}$ if and only if either $u \in X$ and $v \in W \cup Y, u=x^{*}$ and $v \in Y, u \in Z$ and $v \in Y_{2}$, or $u, v \in Y_{1} \cup Y_{2}$. That is, $Y$ is a clique on $t-1$ vertices and $X \cup\left\{x^{*}\right\}, W$ and $Z$ are all independent sets, $H_{n}\left[X \cup\left\{x^{*}\right\}, Y\right]$ is a complete bipartite, and all vertices in $W \cup Z$ have degree $s-1$.

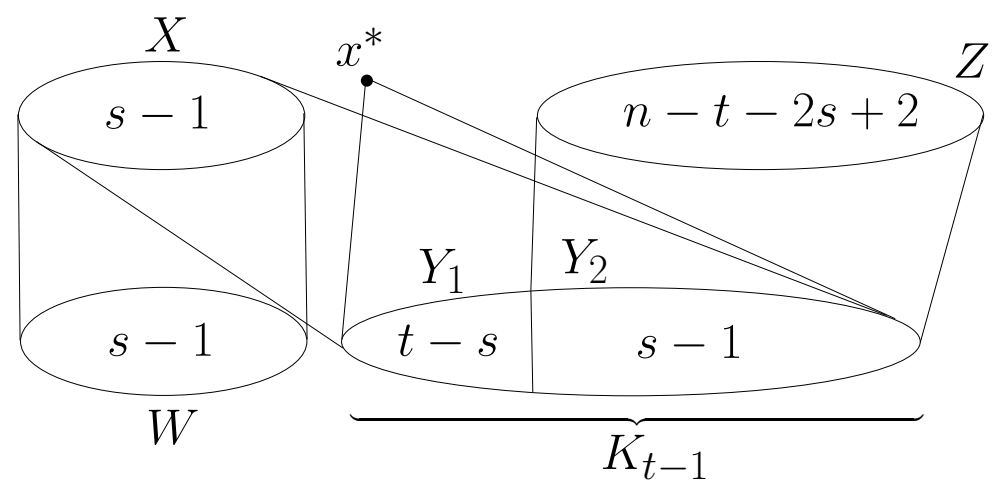

Figure 2: The graph $H_{n}$. The sets $X, W$ and $Z$ are independent sets of cardinalities $s-1, s-1$ and $n-t-2 s+2$, respectively. All edges between $X$ and $W \cup Y_{1} \cup Y_{2}$, between $x^{*}$ and $Y_{1} \cup Y_{2}$ and between $Y_{2}$ and $Z$ are present. Moreover, $Y_{1} \cup Y_{2}$ is a clique on $t-1$ vertices.

The upper bound follows from showing that $H_{n}$ is weakly $\left(K_{n}, K_{s, t}\right)$-saturated. 
Proposition 14. Let $t>s \geq 2$ and $n \geq 2(s+t)-3$. Then

$$
\operatorname{wsat}\left(n, K_{s, t}\right) \leq(s-1)(n-s)+\left(\begin{array}{l}
t \\
2
\end{array}\right) .
$$

Proof. Observe that $H_{n}$ is $K_{s, t}$-free. Indeed, every vertex in $W \cup Z$ has degree $s-1$, and thus none of them are present in a copy of $K_{s, t}$ (as $\left.s<t\right)$. Now look at the subgraph $H_{n}(X, Y)$ obtained by removing these vertices. This graph has $s+t-1$ vertices and thus contains no copy of $K_{s, t}$. We will show that $H_{n}$ is weakly $\left(K_{n}, K_{s, t}\right)$-saturated by adding the edges of $E\left(K_{n}\right) \backslash E\left(H_{n}\right)$ in such a way that the addition of each edge $e$ creates a copy of $K_{s, t}$ containing $e$.

We are first able to add all edges between $x^{*}$ and $W$. Indeed, consider $e=x^{*} w$ for some $w \in W$. Observe that $H_{n}\left[X \cup\left\{x^{*}\right\}, Y \cup\{w\}\right]$ is a copy of $K_{s, t} \backslash\{e\}$ and hence $e$ can be added.

We next show that we can add all edges between $Y_{1}$ and $Z$. Consider $e=y z$, where $y \in Y_{1}$ and $z \in Z$. Observe that $\left|Y_{2} \cup\{y\}\right|=s$ and $\left.\mid\left(Y_{1} \backslash\{y\}\right) \cup X \cup\left\{x^{*}, z\right\}\right) \mid=t$ and thus $H_{n}\left[Y_{2} \cup\{y\},\left(Y_{1} \backslash\right.\right.$ $\left.\{y\}) \cup X \cup\left\{x^{*}, z\right\}\right]$ is a copy of $K_{s, t} \backslash\{e\}$. Hence $e$ can be added.

We can now add all edges between $W$ and $Z$. Let $e=w z$, where $z \in Z$ and $w \in W$. Then $|X \cup\{z\}|=s$ and $|Y \cup\{w\}|=t$, and thus $H_{n}[X \cup\{z\}, Y \cup\{w\}]$ is a copy of $K_{s, t} \backslash\{e\}$. Hence $e$ can be added.

For the next step, we show that we can add all edges between $Y$ and $W$. Let $e=w y$ where $w \in W$ and $y \in Y$. Let $\tilde{Y}$ be a subset of $Y \backslash\{y\}$ of size $s-1$, and $\tilde{Z}$ a subset of $Z$ of size $t-1$. Then $|\tilde{Y} \cup\{w\}|=s$ and $|\tilde{Z} \cup\{y\}|=t$, and thus $H_{n}[\tilde{Y} \cup\{w\}, \tilde{Z} \cup\{y\}]$ is a copy of $K_{s, t} \backslash\{e\}$. Hence $e$ can be added.

Next we can add all edges within $W$. Let $e=u v$, where $u, v \in W$ and let $Z^{\prime}$ be a subset of $Z$ of size $s-1$. Observe that $H_{n}\left[Z^{\prime} \cup\{u\}, Y \cup\{v\}\right]$ is a copy of $K_{s, t} \backslash\{e\}$. Hence $e$ can be added.

It remains to show that any edge $e=u v$, where $u, v \in X \cup\left\{x^{*}\right\} \cup Z$ can be added. As all edges outside of $X \cup\left\{x^{*}\right\} \cup Z$ have been added, and $|Y \cup W|=s+t-2$, then $H_{n}[W \cup\{u\}, Y \cup\{v\}]$ is a copy of $K_{s, t} \backslash\{e\}$. This completes the proof.

For a lower bound for wsat $\left(K_{n}, K_{s, t}\right)$, inspired by the argument in [17] (see also Appendix A), we can show the following.

Proposition 15. Let $t>s \geq 2$ and $n \geq 3 t-3$. Then

$$
\operatorname{wsat}\left(n, K_{s, t}\right) \geq(s-1)(n-t+1)+\left(\begin{array}{l}
t \\
2
\end{array}\right) .
$$

Proof. Let $G \subseteq K_{n}$ be a graph on $n$ vertices, with wsat $\left(K_{n}, K_{s, t}\right)$ edges, and assume that $G$ is weakly $\left(K_{n}, K_{s, t}\right)$-saturated with the corresponding ordering of the missing edges $\left\{e_{1}, \ldots, e_{h}\right\}$ and such that $C_{i}$ is a copy of $K_{s, t}$ created by adding $e_{i}$. Let $G^{\prime}$ be a graph obtained by $G$ as follows. $V\left(G^{\prime}\right)=V(G) \cup X$, where $X$ is a set of size $t-s$ disjoint from $V(G)$, and $E\left(G^{\prime}\right)=E(G) \cup\{v u \mid$ $v \in X, u \in V(G)\}$. Then $G^{\prime}$ is a graph with $n+t-s$ vertices and has $\operatorname{wsat}\left(K_{n}, K_{s, t}\right)+n(t-s)$ edges. Now, note that $G^{\prime}$ is weakly $\left(K_{n+t-s}, K_{t, t}\right)$-saturated. Indeed, the edges $e_{i}, 1 \leq i \leq h$, can still be added using $C_{i}$ together with the $t-s$ new vertices that were added. The edges inside $X$ can then be added using any $2 t-2$ vertices from $V(G)$. By the minimality of wsat, we have that $\left|E\left(G^{\prime}\right)\right| \geq \operatorname{wsat}\left(K_{n+t-s}, K_{t, t}\right)=(t-1)(n-s+1)+\left(\begin{array}{c}t \\ 2\end{array}\right)$ (by Theorem 2). All together, we obtain $\operatorname{wsat}\left(K_{n}, K_{s, t}\right)+n(t-s) \geq(t-1)(n-s+1)+\left(\begin{array}{l}t \\ 2\end{array}\right)$, that is, wsat $\left(K_{n}, K_{s, t}\right) \geq(n-t+1)(s-1)+\left(\begin{array}{l}t \\ 2\end{array}\right)$. 
Note that this lower bound matches the upper bound from Proposition 14 when $s=t-1$, which is the content of Corollary 3. It is also worth mentioning that the upper and lower bounds differ only by $(t-s-1)(s-1)$, and thus Proposition 14 and Proposition 15 imply Theorem 4 .

\section{Conclusion}

In this paper, for $n \geq 3 t-3$ we have exactly determined $\operatorname{wsat}\left(n, K_{t, t}\right)$ (see Theorem 2 ) and $\operatorname{wsat}\left(n, K_{t, t+1}\right)$ (see Corollary 3). Now that $\operatorname{wsat}\left(n, K_{t}\right)$ and wsat $\left(n, K_{t, t}\right)$ are known, the next natural question is to consider balanced multipartite graphs. Let $K_{t}^{k}$ denote the complete multipartite graph containing $k$ parts each of size $t$. A generalisation of our construction for wsat $\left(n, K_{t, t}\right)$ yields a plausibly tight upper bound for wsat $\left(n, K_{t}^{k}\right)$. We are curious as to whether this could be best possible for large $n$ (note that for our constructions to be weakly saturated we need a lower bound on $n)$.

Construction 3. Let $F_{n}^{k, t}$ be an $n$-vertex graph on vertex set $X \cup Y \cup Z$, where $X \cup Y$ contains a complete $k$ partite graph with vertex classes $X=C_{1}, Y=C_{2}, \ldots, C_{k}$ where $\left|C_{i}\right|=t$ for $i \leq k-1$ and $\left|C_{k}\right|=t-1 ; X$ induces a clique on $t$ vertices; and, $Z$ is an independent set of size $n-t k+1$ such that $F_{n}^{k, t}[Y, Z]$ is a complete bipartite graph.

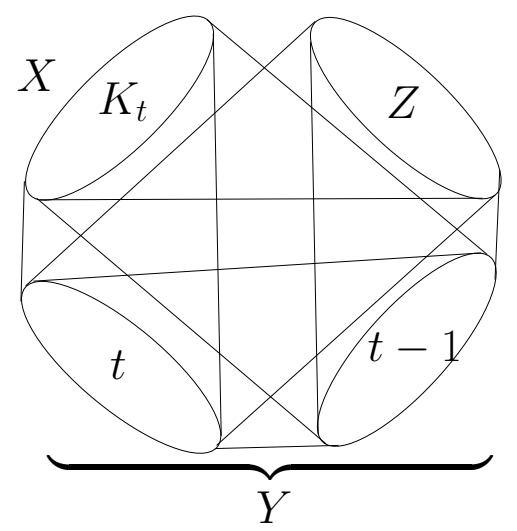

Figure 3: The graph $F_{n}^{3, t}$. The set $X$ is a clique on $t$ vertices while $Z$ is an independent set on $n-3 t+1$ vertices. Additionally, $Y$ induces a complete bipartite graph on vertex classes of sizes $t$ and $t-1$. Every edge between $Y$ and $X \cup Z$ is present.

It is not difficult to check that the graph $F_{n}^{k, t}$ is $K_{t}^{k}$-weakly-saturated (the details are left to the reader) which implies wsat $\left(K_{n}, K_{t}^{k}\right) \leq\left|E\left(F_{n}^{k, t}\right)\right|$ for $n \geq(k+1) t-2$. Observe that $K_{r}$ is isomorphic to $K_{1}^{r}$ and so, by Theorem 1, we have wsat $\left(K_{n}, K_{r}\right)=\left|E\left(F_{n}^{r, 1}\right)\right|$.

Question 1. Is there $n_{0}$ such that for all $n \geq n_{0}$, we have wsat $\left(K_{n}, K_{t}^{k}\right)=\left|E\left(F_{n}^{k, t}\right)\right|$ ?

Let us now turn our attention to unbalanced bipartite graphs. For $2 \leq s<t$ we have provided an asymptotically tight bound on $\operatorname{wsat}\left(n, K_{s, t}\right.$ ) (see Theorem 4). It would be interesting to pin this value down precisely. We wonder if Construction 2 is best possible for large $n$, i.e. if Proposition 14 is tight for large $n$. 
Question 2. Is there some $n_{0}$ such that for all $n \geq n_{0}$ and $t>s+1 \geq 2$, we have

$$
\operatorname{wsat}\left(n, K_{s, t}\right)=(s-1)(n-s)+\left(\begin{array}{l}
t \\
2
\end{array}\right) ?
$$

In particular, we believe that the lower bound given by Proposition 15 is not tight in general; it seems that by analysing the process more carefully it could be possible to add fewer extra edges to convert the $K_{s, t}$ process into the $K_{t, t}$ process.

Although Corollary 8 reveals a relationship between $\operatorname{wsat}\left(n, K_{t}\right)$ and $\operatorname{wsat}\left(K_{\ell, m}, K_{t, t}\right)$, it is not obvious how to use knowledge of the weak saturation numbers of $K_{s, t}$ within $K_{\ell, m}$ to bound $\operatorname{wsat}\left(n, K_{s, t}\right)$. It is plausible (but wrong) to believe that for $s<t$, we have $\operatorname{wsat}\left(n, K_{s, t}\right)>$ $\operatorname{wsat}\left(K_{\ell, m}, K_{s, t}\right)$ (where $\ell+m=n$ ). In $K_{n}$, the process must eventually add more edges (those not respecting the bipartition), but we also have more freedom to choose the edges of the "starting" graph, and it is not obvious that we cannot "save" some edges.

We would like to emphasize that comparing the bounds given by Proposition 14 and Proposition 15 for $\operatorname{wsat}\left(n, K_{s, t}\right)$ to the value of $\operatorname{wsat}\left(K_{\ell, m}, K_{s, t}\right)$ (given by Theorem 7) where $\ell+m=n$, shows that $\operatorname{wsat}(F, H)$ does not obviously compare to wsat $\left(F^{\prime}, H\right)$ when $F^{\prime} \subseteq F$. Indeed, by Proposition 14, when $t>3 s$ we get $\operatorname{wsat}\left(K_{n}, K_{s, t}\right)<\operatorname{wsat}\left(K_{\ell, m}, K_{s, t}\right)$, and by Proposition 15, for $2 s>t$ we get $\operatorname{wsat}\left(K_{n}, K_{s, t}\right) \geq \operatorname{wsat}\left(K_{\ell, m}, K_{s, t}\right)$.

We therefore believe it is interesting to consider the following.

Question 3. Let $2 \leq s<t$ and $k+\ell=n$, for large $n$. When do we have

$$
\operatorname{wsat}\left(n, K_{s, t}\right)>\operatorname{wsat}\left(K_{\ell, m}, K_{s, t}\right) ?
$$

It would also be very interesting to determine weak saturation numbers of general unbalanced multipartite graphs. Let $K_{a_{1}, \ldots, a_{k}}$ denote the complete multipartite graph with parts of size $a_{1}, \ldots, a_{k}$.

Question 4. What is $\operatorname{wsat}\left(n, K_{a_{1}, \ldots, a_{k}}\right)$ ?

We remark that for $a_{1}, \ldots, a_{k-1} \leq a_{k}$, an argument analogous to the one in Proposition[15]would give a lower bound on $\operatorname{wsat}\left(n, K_{a_{1}, \ldots, a_{k}}\right)$ based on $\operatorname{wsat}\left(n+\left(a_{k}-a_{k-1}\right)+\ldots+\left(a_{k}-a_{1}\right), K_{a_{k}, \ldots, a_{k}}\right)$. In general, wsat $\left(n, K_{a_{1}, \ldots, a_{k}}\right)$ can always be lower bounded based on $\operatorname{wsat}\left(n+\left(s_{1}-a_{1}\right)+\ldots+\left(s_{k}-\right.\right.$ $\left.\left.a_{k}\right), K_{s_{1}, \ldots, s_{k}}\right)$ as long as $a_{i} \leq s_{i}$ for $i \in[k]$. Although this method could give a good lower bound for an asymptotic result, we do not believe that it would give a tight bound.

\section{Acknowledgment}

This work began while the authors were visiting the Instituto Nacional de Matemática Pura e Aplicada in Rio de Janeiro as part of the Graphs@IMPA thematic program. We are very grateful to IMPA and the organizers for the support to be able to attend the event and the great working environment. 


\section{References}

[1] N. Alon: An extremal problem for sets with applications to graph theory, J. Combin. Theory Ser. A 40 (1985), 82-89.

[2] J. Balogh, B. Bollobás, R. Morris and O. Riordan: Linear algebra and bootstrap percolation, J. Combin. Theory Ser. A 119 (2012), 1328-1335.

[3] J. Balogh and G. Pete: Random disease on the square grid, Random Structures Algorithms 13 (1998), 409-422.

[4] B. Bollobás: On generalized graphs, Acta Math. 16 (1965), 447-452.

[5] B. Bollobás: Weakly k-saturated graphs, Beiträge zur Graphentheorie (1968) 25-31.

[6] M. Borowiecki and E. Sidorowicz: Weakly P-saturated graphs, Discuss. Math. Graph Theory 22 (2002), 17-29.

[7] P. Erdős, Füredi and Z. Tuza: Saturated r-uniform hypergraphs, Discrete Math. 98 (1991), 95-104.

[8] J. R. Faudree, R. J. Faudree and J. R. Schmitt: A survey of minimum saturated graphs, Electron. J. Comb. 1000 (2011), 19-29.

[9] R. J. Faudree and R. J. Gould: Weak saturation numbers for multiple copies, Discrete Math. 336 (2014), 1-6.

[10] R. J. Faudree, R. J. Gould and M. S. Jacobson: Weak saturation numbers for sparse graphs Discuss. Math. Graph Theory 33 (2013), 677-693.

[11] P. Frankl: An extremal problem for two families of sets, European J. Combin. 3 (1982), 125127.

[12] G. Kalai: Hyperconnectivity of graphs, Graphs Combin. 1 (1985), 65-79.

[13] G. Kalai: Weakly saturated graphs are rigid, North-Holland Math. Stud. 87 (1984), 189-190.

[14] L. Lovász: Flats in matroids and geometric graphs, Combinatorial Surveys (1977), 45-86.

[15] N. Morrison and J. A. Noel: A Sharp Threshold for Bootstrap Percolation in a Random Hypergraph, available as arXiv:1806.02903.

[16] N. Morrison and J. A. Noel: Extremal bounds for bootstrap percolation in the hypercube, J. Combin. Theory Ser. A 156 (2018), 61-84.

[17] G. Moshkovitz and A. Shapira: Exact bounds for some hypergraph saturation problems, J. Combin. Theory Ser. B 111 (2015), 242-248.

[18] O. Pikhurko: Uniform families and count matroids, Graphs Combin. 17 (2001), 729-740. 
[19] O. Pikhurko: Weakly saturated hypergraphs and exterior algebra, Combin. Probab. Comput. 10 (2001), 435-451.

[20] G. Semanišin: On some variations of extremal graph problems, Discuss. Math. Graph Theory 17 (1997), 67-76.

[21] E. Sidorowicz: Size of weakly saturated graphs, Discrete Math. 307 (2007), 1486-1492.

[22] Z. Tuza: Asymptotic growth of sparse saturated structures is locally determined, Discrete Math. 108 (1992), 397-402.

[23] Z. Tuza: Extremal problems on saturated graphs and hypergraphs, Ars Combin. 25 (1988), 105-113.

\section{A Proof of Theorem 7}

Here we will prove Theorem 7 by generalising the argument from [17] for Theorem 6 .

Proof of Theorem 7 . We start with the upper bound. Let $X_{1}, X_{2}, X_{3}, Y_{1}, Y_{2}, Y_{3}$ be disjoint sets of vertices such that $\left|X_{1}\right|=\left|Y_{1}\right|=s-1,\left|X_{2}\right|=\left|Y_{2}\right|=t-s,\left|X_{3}\right|=\ell-t+1,\left|Y_{3}\right|=m-t+1$. Denote $X=X_{1} \cup X_{2} \cup X_{3}$ and $Y=Y_{1} \cup Y_{2} \cup Y_{3}$. Note that $|X|=\ell$ and $|Y|=m$. Let $G_{0} \subseteq K_{\ell, m}$ be a bipartite graph on vertex set $X \cup Y$ where $v u \in E\left(G_{0}\right)$ if and only if $v \in X_{1}$ and $u \in Y$, or $v \in Y_{1}$ and $u \in X$, or $v \in X_{2}$ and $u \in Y_{2}$. Note that $\left|E\left(G_{0}\right)\right|=(\ell+m)(s-1)+(t-s)^{2}$. We will show that $G_{0}$ is weakly $\left(K_{\ell, m}, K_{s, t}\right)$-saturated.

Since $G_{0}\left[X_{1} \cup X_{2}, Y_{1} \cup Y_{2}\right], G_{0}\left[X, Y_{3}\right]$ and $G_{0}\left[X_{3}, Y\right]$ are all complete bipartite graphs, we only need to show how to add the edges $v u$ when $v \in X_{3}$ and $u \in Y_{2} \cup Y_{3}$, and when $v \in Y_{3}$ and $u \in X_{2}$.

We start with adding the edges $v u$ when $v \in Y_{3}$ and $u \in X_{2}$. Since $\left|\{u\} \cup X_{1}\right|=s, \mid\{v\} \cup$ $X_{1} \cup X_{2} \mid=t$, and $G_{0}\left[\{u\} \cup X_{1},\{v\} \cup Y_{1} \cup Y_{2}\right]$ is a copy of $K_{s, t}$ minus one edge, we can add the missing edge $v u$. In a similar fashion, we can add edges $v u$ when $v \in Y_{2}$ and $u \in X_{3}$. Now we only need to add the edges $v u$ for $v \in X_{3}$ and $u \in Y_{3}$. Since $\left|\{v\} \cup X_{1}\right|=s,\left|\{u\} \cup Y_{1} \cup Y_{2}\right|=t$, and $G_{0}\left[\{v\} \cup X_{1},\{u\} \cup Y_{1} \cup Y_{2}\right]$ is a copy of $K_{s, t}$ missing $v u$, we can add the edge $v u$. This shows that $G_{0}$ is weakly $\left(K_{\ell, m}, K_{s, t}\right)$-saturated and completes the proof of the upper bound.

For the lower bound, we will apply Theorem 6. Let $G \subseteq K_{\ell, m}$ be a weakly $\left(K_{\ell, m}, K_{s, t}\right)$-saturated graph with wsat $\left(K_{\ell, m}, K_{s, t}\right)$ edges. Let $\left\{e_{1}, \ldots, e_{h}\right\}$ be an ordering of $E\left(K_{\ell, m} \backslash G\right)$ such that $C_{i}$ is a copy of $K_{s, t}$ in $G \cup\left\{e_{1}, \ldots, e_{i}\right\}$ containing $e_{i}$.

Denote by $X_{1}$ the vertex set of $G$ of size $\ell$, and by $Y_{1}$ the vertex set of $G$ of size $m$. Let $X_{2}, Y_{2}$ be two disjoint sets (also disjoint from $X_{1}, Y_{1}$ ), each of size $t-s$. Let $G^{\prime}$ be the bipartite graph with parts $X=X_{1} \cup X_{2}$ and $Y=Y_{1} \cup Y_{2}$, obtained from $G$ as follows. $E\left(G^{\prime}\right)=E(G) \cup\{v u \mid$ $\left.v \in X_{2}, u \in Y_{1}\right\} \cup\left\{v u \mid v \in Y_{2}, u \in X_{1}\right\}$. Then $G^{\prime}$ is a bipartite graph with parts of size $\ell+t-s$ and $m+t-s$, and has $\operatorname{wsat}\left(K_{\ell, m}, K_{s, t}\right)+(\ell+m)(t-s)$ edges. Now, note that $G^{\prime}$ is weakly $\left(K_{\ell+t-s, m+t-s}, K_{t, t}\right)$-saturated. Indeed, the edges $e_{i}, 1 \leq i \leq h$, can still be added using $C_{i}$ together with the $t-s$ new vertices that were added to one of the sides. The edges between $X_{2}$ and 
$Y_{2}$ can then be added using $t-1$ vertices from $X_{1}$ and $t-1$ vertices from $Y_{1}$. By the minimality of wsat, we have that

$$
\left|E\left(G^{\prime}\right)\right| \geq \operatorname{wsat}\left(K_{\ell+t-s, m+t-s}, K_{t, t}\right)=(\ell+t-s)(m+t-s)-(\ell-s+1)(m-s+1) .
$$

As $\left|E\left(G^{\prime}\right)\right|=\operatorname{wsat}\left(K_{\ell, m}, K_{s, t}\right)+(\ell+m)(t-s)$, we obtain

$$
\operatorname{wsat}\left(K_{\ell, m}, K_{s, t}\right) \geq \ell m-(\ell+t-s)(m+t-s)+(t-s)^{2},
$$

as required. 